\title{
Pairing-up SOX to kick-start beta cell genesis
}

\author{
Anne Grapin-Botton • Philip Allan Seymour • \\ Gérard Gradwohl
}

Received: 3 February 2015 / Accepted: 6 February 2015 / Published online: 4 March 2015

(C) Springer-Verlag Berlin Heidelberg 2015

\begin{abstract}
The transcription factor SOX9 is regarded as a crucial player in pancreas development, both maintaining progenitors and later being required for beta cell differentiation. However, very little is known about the possible involvement of other SOX family members in such processes. In this issue, the work of Xu et al (DOI: 10.1007/s00125-015-3507-x) shines a spotlight on SOX4, revealing this factor to be a major player in the beta cell program. Using conditional inactivation in mice, they show that SOX4 shares some functions in progenitors with SOX9, but also plays a distinct role at a later stage of development, during the maturation of endocrine cells. This information is timely as this final maturation process is currently the most challenging to reproduce in vitro when coaxing pluripotent stem cells to convert into beta cells.
\end{abstract}

Keywords Beta cell · Development - Diabetes · Differentiation - Embryo - Endocrine cell - Mouse . Progenitors - Stem cells $\cdot$ Transcription factors

\begin{tabular}{ll}
\multicolumn{2}{l}{ Abbreviations } \\
E & Embryonic day \\
hPSC & Human pluripotent stem cell \\
ISL1 & ISL LIM homeobox 1 \\
NEUROG3 & Neurogenin 3 \\
RFX6 & Regulatory factor X, 6
\end{tabular}

A. Grapin-Botton $(\square) \cdot$ P. A. Seymour

DanStem, University of Copenhagen, 3B Blegdamsvej,

DK-2200 Copenhagen N, Denmark

e-mail: Anne.grapin-botton@sund.ku.dk

G. Gradwohl

Institut de Génétique et de Biologie Moléculaire et Cellulaire, Institut

National de la Santé et de la Recherche Médicale U964, Centre

National de Recherche Scientifique UMR7104, Université de

Strasbourg, Illkirch 67404, France $\begin{array}{ll}\text { SOX4 } & \begin{array}{l}\text { Sex-determining region Y-related high } \\ \text { mobility group box } 4 \\ \text { TF }\end{array} \\ \text { Transcription factor }\end{array}$

There are two major medical reasons to study the development of the pancreas and its endocrine cells. The first is that conditions and diseases that only manifest postnatally or even in the adult sometimes stem from embryonic developmental defects. In this regard, diabetes is certainly no exception. While fetal programming of diabetes and neonatal diabetes are obvious examples $[1,2]$, this may extend to other forms of type 1 or type 2 diabetes. The second reason for studying pancreatic development is that understanding how beta cells are formed in the embryo provides a roadmap to produce them in vitro from human pluripotent stem cells (hPSCs). While the ability to produce beta cells in potentially unlimited numbers holds great allure as a prospective diabetes therapy, in vitro-generated beta cells are significant in their own right as models of human beta cells, be that normal or genetically impaired [3].

Beta cells, like the other specialised cells in the pancreas, arise from self-renewing progenitors that either differentiate into the various pancreatic cell types or divide to yield new progenitors, so expanding the progenitor pool to enable continued growth of the organ. Over the course of development, the ability of progenitors to form different specialised pancreatic cell types (their 'potency') progressively narrows. This restriction of potency is a stepwise process, the primogenitor being the 'multipotent' pancreatic progenitor that is capable of generating all of the specialised cells of the pancreas, but not those of other organs (Fig. 1). This cell type is transient and current evidence suggests that it only exists at the earliest stages of pancreas development (embryonic day [E]8.5E13.5 in mice) [4]. These multipotent progenitors then give rise to either specialised acinar-specific progenitors or socalled 'bipotent' progenitors that yield the ductal cells and 


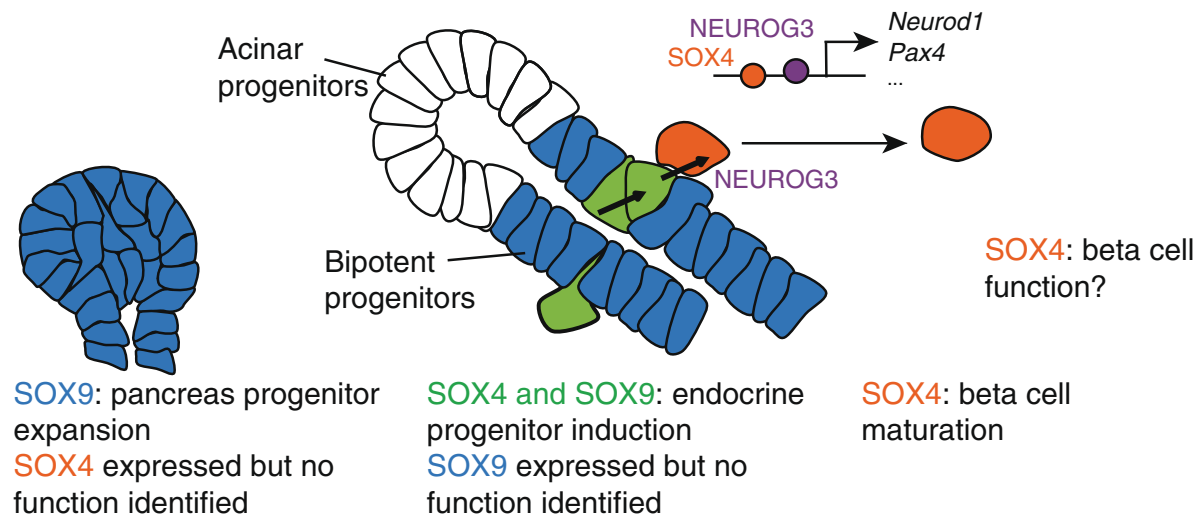

\section{Pancreas bud, E10.5 Pancreas tree, E13.5-E17.5 Postnatally}

Fig. 1 Summary of SOX4 and SOX9 functions during development and possible adult function of SOX4. The stages of development are represented from left to right: pancreas bud (mouse E10.5), pancreas tree (mouse E13.5-E17.5), adult beta cell. The colour code represents a combination of expression and activity of SOX4 (orange), SOX9 (blue) or both (green) in different cell types. The endocrine progenitors expressing NEUROG3 (green cells) are represented as exiting the ductal epithelium and becoming endocrine cells (orange) in the middle panel. The schematic above represents direct binding and activity of NEUROG3 and SOX4 on Neurodl and Pax4 promoters endocrine cells of the islets (Fig. 1). The latter, in turn, arise via an endocrine progenitor intermediate, derived from pancreatic or bipotent progenitors (Fig. 1). Such endocrine progenitors are transient and generally non-proliferative, each differentiating into a single endocrine cell.

In this issue of Diabetologia, Xu et al [5] demonstrate that the transcription factor $(\mathrm{TF})$ sex-determining region Y-related high mobility group box 4 (SOX4) controls the generation of beta cells at two distinct developmental steps: first, the production of neurogenin 3 (NEUROG3)-expressing endocrine progenitors and then, second, their subsequent differentiation into endocrine cells. This role is somewhat unexpected in that it differs from that of another SOX-family protein, SOX9, which plays a major role in the maintenance and expansion of multipotent pancreatic progenitors. While also expressed in such progenitors, SOX4 is apparently not required for their maintenance in mice. In spite of these differences, SOX9 and SOX4 both promote endocrine differentiation in progenitors by directly upregulating Neurog 3 expression via its promoter [6-8]. The relatively modest reduction $(30 \%)$ in endocrine progenitor numbers upon Sox4 inactivation in pancreas progenitors implies functional redundancy between SOX4 and possibly other family members. The often parallel roles played by members of the same subgroup [9] make intra-subgroup redundancy more likely. Thus, it is plausible that absence of SOX4 is partially compensated by, for example, SOX11, which, like SOX4, is a subgroup C factor ostensibly expressed in developing endocrine cells [10] and has been shown to be upregulated in Sox4-null pancreases [11]. Given their colocalisation in a subpopulation of progenitors, it is also possible that SOX9 and SOX4 are redundant in initiating endocrine commitment. This is, however, their only functional redundancy, as SOX4 is expressed in nascent endocrine cells and promotes their maturation into beta cells, a function not shared with SOX9. This feature places SOX4 in the limelight, as the final maturation of beta cells is currently the most limiting step in the hPSC-to-beta cell pipeline, in spite of considerable recent advances made in this endeavour $[12,13]$. Xu et al [5] show that if Sox4 is deleted in Neurog3-expressing endocrine progenitors in mice, the endocrine cells, instead of differentiating, accumulate as an apparently generic secretory cell expressing synaptophysin and chromogranin $\mathrm{A}$. While the exact identity and differentiation status of these partially differentiated cells remains unclear, what is clear is that the expression of crucial endocrine TFs acting atop a hierarchy of functional genes is greatly reduced. This is the case, for example, for NeuroD and Pax4: their expression is positively and synergistically regulated by direct co-binding of NEUROG3 and SOX4. This feature is a characteristic of SOX proteins, which usually exert their transcriptional activity by forming complexes with other transcription factors, implying that SOX binding sites in target genes are in the vicinity of a binding site for the partner protein. However, it remains to be demonstrated whether SOX4 and NEUROG3 TFs physically interact to promote the transactivation of NeuroD and Pax4 (or other genes) during endocrine differentiation. Importantly, the dual function of SOX4 revealed by $\mathrm{Xu}$ et al [5], namely, the promotion of endocrine commitment and subsequent maturation of nascent endocrine cells, could potentially be explained by substitution of SOX4 partners. This would result in a dramatic change in the repertoire of target genes. One can speculate that the SOX4-TF complex activates the transcription of another transcription factor, which then functions as a novel partner. Alternatively, SOX factor exchange (e.g. SOX4 for SOX9), while the partner remains the same, could be conceived as well. Both 
mechanisms have been demonstrated to promote the stepwise progression of developmental processes in other paradigms (see [14] for a review). Xu et al now possess the tools to further decipher the SOX4-associated transcriptional machinery governing endocrine development.

This partial differentiation phenotype, characterised by islet cells with generic hallmarks of endocrine cells but lacking hormones, is reminiscent of that seen upon loss of the endocrine TFs insulinoma-associated 1 (INSM1), ISL LIM homeobox 1 (ISL1) or regulatory factor X, 6 (RFX6) [15-18]. These factors are likely to synergise in a TF network promoting beta cell maturation. Sox4 has not yet been scrutinised in the quality control of hPSC-to-beta cell differentiation protocols and appears to deserve more attention. Whether SOX4 plays a similarly significant role in beta cell development in humans as in the mouse remains to be seen.

While a previous report in zebrafish [19] already implicated a crucial role for Sox 4 in endocrine differentiation, its role in this species appears to be restricted to glucagon-producing cells. However, this may be due to the fact that this study did not examine the role of Sox 4 in the generation of the late beta cells, which most resemble those of mammals. Alternatively, the divergence in Sox4-deficient pancreatic phenotypes between mouse and zebrafish may reflect discrepancies in the transcriptional programs governing endocrine differentiation in these two models.

As is the case for several pancreatic TFs (e.g. pancreatic and duodenal homeobox 1 [PDX1], RFX6, neurogenic differentiation 1 [NEUROD1], NK6 homeobox 1 [NKX6.1], ISL1), SOX4 appears to be an essential factor both for beta cell development and postnatal function. The latter role is supported by the study of two independent autosomaldominant $N$-ethyl- $N$-nitrosourea (ENU)-induced mutations in the SOX4 high mobility group box, leading to glucose intolerance and impaired insulin secretion in mice [20]. However, it cannot be completely excluded that such mice carry additional ENU mutations that might contribute to this phenotype. Thus, the generation and examination of mice lacking Sox4 specifically in beta cells will now enable the confirmation and further dissection of the role of SOX4 in beta cell function.

Duality of interest The authors declare that there is no duality of interest associated with this manuscript.

Contribution statement All authors were equally responsible for the conception, design and drafting of the manuscript, and approved the final version for publication.

\section{References}

1. Flanagan SE, De Franco E, Lango Allen H et al (2014) Analysis of transcription factors key for mouse pancreatic development establishes NKX2-2 and MNX1 mutations as causes of neonatal diabetes in man. Cell Metab 19:146-154

2. Yajnik CS (2013) Commentary: thrifty phenotype: 20 years later. Int J Epidemiol 42:1227-1229

3. Abdelalim EM, Bonnefond A, Bennaceur-Griscelli A, Froguel P (2014) Pluripotent stem cells as a potential tool for disease modelling and cell therapy in diabetes. Stem Cell Rev 10:327-337

4. Pan FC, Wright C (2011) Pancreas organogenesis: from bud to plexus to gland. Dev Dyn 240:530-565

5. Xu EE, Krentz NA, Tan S et al (2015) SOX4 cooperates with neurogenin 3 to regulate endocrine pancreas formation in mouse models. Diabetologia. doi:10.1007/s00125-015-3507-x

6. Lynn FC, Smith SB, Wilson ME, Yang KY, Nekrep N, German MS (2007) Sox9 coordinates a transcriptional network in pancreatic progenitor cells. Proc Natl Acad Sci U S A 104:10500-10505

7. Seymour PA, Freude KK, Dubois CL, Shih HP, Patel NA, Sander M (2008) A dosage-dependent requirement for Sox9 in pancreatic endocrine cell formation. Dev Biol 323:19-30

8. Shih HP, Kopp JL, Sandhu M et al (2012) A Notch-dependent molecular circuitry initiates pancreatic endocrine and ductal cell differentiation. Development 139:2488-2499

9. Wegner M (2010) All purpose Sox: the many roles of Sox proteins in gene expression. Int J Biochem Cell Biol 42:381-390

10. Lioubinski O, Muller M, Wegner M, Sander M (2003) Expression of Sox transcription factors in the developing mouse pancreas. Dev Dyn 227:402-408

11. Wilson ME, Yang KY, Kalousova A et al (2005) The HMG box transcription factor Sox 4 contributes to the development of the endocrine pancreas. Diabetes 54:3402-3409

12. Pagliuca FW, Millman JR, Gurtler M et al (2014) Generation of functional human pancreatic beta cells in vitro. Cell 159:428-439

13. Rezania A, Bruin JE, Arora P et al (2014) Reversal of diabetes with insulin-producing cells derived in vitro from human pluripotent stem cells. Nat Biotechnol 32:1121-1133

14. Kamachi Y, Kondoh H (2013) Sox proteins: regulators of cell fate specification and differentiation. Development 140:4129-4144

15. Gierl MS, Karoulias N, Wende H, Strehle M, Birchmeier C (2006) The zinc-finger factor Insm1 (IA-1) is essential for the development of pancreatic beta cells and intestinal endocrine cells. Genes Dev 20: 2465-2478

16. Du A, Hunter CS, Murray J et al (2009) Islet-1 is required for the maturation, proliferation, and survival of the endocrine pancreas. Diabetes 58:2059-2069

17. Smith SB, Qu HQ, Taleb N et al (2010) Rfx6 directs islet formation and insulin production in mice and humans. Nature 463:775-780

18. Piccand J, Strasser P, Hodson DJ et al (2014) Rfx6 maintains the functional identity of adult pancreatic beta cells. Cell Rep 9:2219 2232

19. Mavropoulos A, Devos N, Biemar F et al (2005) sox4b is a key player of pancreatic alpha cell differentiation in zebrafish. Dev Biol 285:211-223

20. Goldsworthy M, Hugill A, Freeman H et al (2008) Role of the transcription factor sox 4 in insulin secretion and impaired glucose tolerance. Diabetes 57:2234-2244 